\title{
Change of Extracellular Glutamate Level in Striatum during Deep Brain Stimulation of the Entopeduncular Nucleus in Rats
}

\author{
Hyun-ju Lee, Ph.D., Jae Hoon Sung, M.D., Ph.D., ${ }^{1}$ Jae Taek Hong, M.D., Ph.D., ${ }^{2}$ I Sup Kim, M.D., Ph.D., ${ }^{2}$ \\ Seung Ho Yang, M.D., Ph.D., ${ }^{2}$ Chul Bum Cho, M.D., Ph.D. ${ }^{2}$ \\ Department of Science in Korean Medicine, Graduate School, College of Korean Medicine, Kyung Hee University, Seoul, Korea \\ Department of Neurosurgery, ${ }^{2}$ St. Vincent's Hospital, College of Medicine, The Catholic University of Korea, Seoul, Korea
}

Objective : Globus pallidus interna (GPi) is acknowledged as an essential treatment for advanced Parkinson's disease (PD). Nonetheless, the neurotransmitter study about its results is undiscovered. The goal of this research was to examine influences of entopeduncular nucleus (EPN) stimulation, identical to human GPi, in no-lesioned (NL) rat and 6-hydroxydopamine (6-HD)-lesioned rat on glutamate change in the striatum.

Methods : Extracellular glutamate level changes in striatum of NL category, NL with deep brain stimulation (DBS) category, 6-HD category, and 6-HD with DBS category were examined using microdialysis and high-pressure liquid chromatography. Tyrosine hydroxylase (TH) immunoreactivities in substantia nigra and striatum of the four categories were also analyzed.

Results : Extracellular glutamate levels in the striatum of NL with DBS category and 6-HD with DBS category were significantly increased by EPN stimulation compared to those in the NL category and 6-HD category. EPN stimulation had no significant effect on the expression of TH in NL or 6-HD category.

Conclusion : Clinical results of GPi DBS are not only limited to direct inhibitory outflow to thalamus. They also include extensive alteration within basal ganglia.

Key Words : Entopeduncular nucleus · Glutamates · Deep brain stimulation · Parkinson disease.

\section{INTRODUCTION}

Parkinson's disease (PD) is a neurodegenerative disease defined as dopamine depletion due to the degeneration of substantia nigra (SN) dopamine neurons. Globus pallidus interna (GPi) stimulation has become an established treatment for motor symptoms of $\mathrm{PD}^{13,21,25,29)}$. However, the exact mechanism of deep brain stimulation (DBS) in GPi remains unrevealed. At cellular level, DBS has complex effects by driving and inhibiting neural components depending on the stimulation parameters and location ${ }^{3,18,25)}$.

Gamma-aminobutyric acid (GABA), glutamate and dopa-

- Received : May 31, 2018 •Revised : July 16, 2018 •Accepted : July 24, 2018

- Address for reprints : Chul Bum Cho, M.D., Ph.D.

Department of Neurosurgery, St. Vincent's Hospital, College of Medicine, The Catholic University of Korea, 93 Jungbu-daero, Paldal-gu, Suwon 16247, Korea Tel : +82-31-249-8929, Fax : +82-31-245-5208, E-mail : medics0919@gmail.com

This is an Open Access article distributed under the terms of the Creative Commons Attribution Non-Commercial License (http://creativecommons.org/licenses/by-nc/4.0) which permits unrestricted non-commercial use, distribution, and reproduction in any medium, provided the original work is properly cited. 
mine are main neurotransmitters in basal ganglia circuit. Although there have been a few papers regarding extracellular striatal glutamate changes by subthalamic nucleus (STN) stimulation ${ }^{5,6,14)}$, extracellular striatal glutamate changes or tyrosine hydroxylase (TH) immunoreactivities after GPi stimulation have not been reported yet.

Entopeduncular nucleus (EPN) of rat corresponds to GPi in humans and primates. Studying changes of extracellular glutamate level in striatum after EPN stimulation may contribute to the knowledge of the influence of glutamate in striatal and nigral dopamine system ${ }^{12}$. Thus, the goal of this research was to compare influences of EPN stimulation on extracellular striatal glutamate levels of NL category, NL with DBS category, 6-hydroxydopamine (6-HD) category, and 6-HD with DBS category.

\section{MATERIALS AND METHODS}

\section{Animals}

Male Sprague-Dawley rats (Samtako Animal Co., Seoul, Korea) aged seven weeks (260-280 g) were housed at 12-hour light/ dark cycle (light from 8 A.M.) with temperature controlled at $23 \pm$ $1^{\circ} \mathrm{C}$ and relative humidity of $50 \pm 5 \%$. Rats were provided free access to food ad libitum and water. They were acclimated for one week before experiments. The care and treatment of animals followed guidelines of the US National Institutes of Health (Guide for the Care and Use of Laboratory Animals, 8th edition, 2011). All experiments were performed after obtaining approval from the Institutional Animal Care and Use Committee of the Kyung Hee University (KHUAP(SE)-14-051).

\section{Experimental design}

Rats were divided into four groups ( $n=6$ per category) : 1) NL category (no treatment), 2) NL with DBS category, 3) 6-HD category (no treatment), and 4) 6-HD with DBS category. To evaluate the potency of 6-HD in inducing Parkinson motor deficits and lesions, rats were checked through apomorphine (Sigma-Aldrich Inc., St. Louis, MO, USA) induced $(0.05 \mathrm{mg} / \mathrm{kg}$, subcutaneous injection) rotational reaction on three weeks following exposure to 6-HD. Results were expressed as ipsilateral turns/min. Rats demonstrating active rotation (above 100 turns during 45 minutes) to apomorphine were chosen for next step experiment. It has previously been demonstrated that rats meeting this criterion have greater than $95 \%$ depletion of dopamine in the striatum ${ }^{22}$.

\section{6-HD induced lesions}

To induce medial forebrain bundle (MFB) lesion, rats in 6 -HD category $(n=6)$ were under anesthesia using sodium pentobarbital (50 mg/kg, intraperitoneal injection) and restricted in a stereotactic instrument (Stoelting Co., Wood Dale, IL, USA). Then $20 \mu \mathrm{g}$ of 6-HD (Sigma-Aldrich Inc.; $4 \mu \mathrm{g} / \mu \mathrm{L}$ in saline solution) was injected into the right MFB using syringe pump (KDS310; KD Scientific, Holliston, MA, USA) at a flow rate of $2 \mu \mathrm{L} / \mathrm{min}$. Stereotactic dimensions of the injection point were : anteroposterior (AP), $-4.4 \mathrm{~mm}$; lateral (L), $-1.2 \mathrm{~mm}$ from the bregma; and dorsoventral (DV), $-7.8 \mathrm{~mm}$ below the dura. All stereotaxic co-ordinates used in this study were based on stereotactic atlas of Paxinos ${ }^{23)}$. Rats were kept warm environment after 6-HD injections and allowed to recover from anesthesia. They were returned to the animal facility and maintained for 21 days to allow dopaminergic neurons degeneration caused by 6-HD. Rats were managed for microdialysis experiments after 21 days.

\section{Surgery and microdialysis procedures}

For microdialysis experiment, rats were in the process of anesthesia using sodium pentobarbital $(50 \mathrm{mg} / \mathrm{kg}$, intraperitoneal injection) at 3 weeks following 6-HD injection into the MFB. Using aseptic technique, a guide cannula (CMA/12; CMA Microdialysis, Solna, Sweden) was tightly screwed into cannula holder attached to the right-side arm of the stereotaxic and implanted to the dorsal striatum (AP, $1.2 \mathrm{~mm}$;,+3.2 $\mathrm{mm}$ from Bregma; DV, -6.0 mm below dura). Microdialysis was done using a $2 \mathrm{~mm}$ microdialysis probe (CMA/12; CMA Microdialysis) linked to a syringe pump (CMA/402; CMA Microdialysis) through double liquid swivel which was installed to the guide cannula that was supplied with an artificial cerebrospinal fluid $\left(150.0 \mathrm{mM} \mathrm{Na}^{+}, 3.0 \mathrm{mM} \mathrm{K}, 1.4 \mathrm{mM}\right.$ $\mathrm{Ca}^{2+}, 0.8 \mathrm{mM} \mathrm{Mg}^{2+}, 1.0 \mathrm{mM} \mathrm{HPO}_{4}{ }^{2-}, 144 \mathrm{mM} \mathrm{Cl}$, $\mathrm{pH} 7.4$; Tocris Bioscience, Bristol, UK) at a constant rate of $1.0 \mu \mathrm{L} / \mathrm{min}$. Dialysate samples were gathered on ice every 20 minutes through outlet polyethylene-10 tubing (Plastic One, CITY, PA, USA). To measure the concentration of L-glutamate samples from the fourth dialysate was analyzed. 


\section{DBS}

Concentric bipolar platinum/iridium stimulating electrodes (style CB-BFE75; FHC, Inc., Bowdoin, ME, USA) were tightly screwed to the electrode holder attached to the left arm of the stereotactic instrument. Stereotactic dimensions of the stimulation site (EPN) were : $\mathrm{AP},-2.52 \mathrm{~mm}$;,$-3.0 \mathrm{~mm}$ from the bregma; and DV, -7.8 mm below the dura. We delivered stimuli for 20 minutes with a stimulus isolation unit and programmable pulse generator (Iso-Flex/Master-8; AMPI, Jerusalem, Israel). Stimulation parameters applicable to those used in clinical setting $(200 \mu \mathrm{A}$ intensity, frequency $130 \mathrm{~Hz}$ and $60 \mu \mathrm{s}$ pulse width).

\section{L-glutamate measurement}

Dialysate samples $(20 \mu \mathrm{L})$ were evaluated through high performance liquid chromatography (HPLC) assay equipped with fluorescence detector. HPLC system consisted of a fluorescence detector (FLD 2475; Waters Corporation, Manchester, UK) coupled to LC-10 AD pump and a chromatograph with $200 \mu \mathrm{L}$ loop (Rpeodyne 7725-I; Merck, Temecula, CA, USA). The fluorescent detector was set at an emission wavelength of $454 \mathrm{~nm}$ and an excitation wavelength of $337 \mathrm{~nm}$. Mobile phase included tetrahydrofuran, 0.05 M sodium acetate, and HPLC grade methanol adjusted into $\mathrm{pH} 4.0$ with acetic acid. Mobile phase was filtered using $0.45 \mu \mathrm{m}$ durapore membrane filters (Millipore, County Cork, Ireland) followed by vacuum degas. It was then provided at a flow rate of $1.0 \mathrm{~mL} / \mathrm{min}$ through C18 analytical column $(150 \times 4.6 \mathrm{~mm}$, ID, Hibar-Futigsanle RT) with particle size of $3 \mu \mathrm{m}$ and a prepacked column (RE 250-4; Merck, Temecula, CA, USA). Sample peak areas were calculated via an integrator system (Empower 2) and evaluated with calibration standard curve to quantify L-glutamate concentration. L-glutamate in dialysate was showed as percentage of three samples collected immediately before DBS.

\section{TH immunohistochemistry}

After microdialysis processes, all rats were deeply anesthetized with sodium pentobarbital $(80 \mathrm{mg} / \mathrm{kg}$, intraperitoneal injection). After transcardial perfusion using 4\% formaldehyde (Sigma-Aldrich Inc.), rat brains were extracted, fixed for 24 hours, and put in PBS including 20\% sucrose for 3 days. Coronal sections ( $30 \mu \mathrm{m}$ in thickness) were obtained with a cryostat microtome (CM1850UV, Leica Microsystems Inc., Wetzlar, Germany). These sections were kept at $-20^{\circ} \mathrm{C}$ for his- tochemical processing as free-floating sections. These sections were cleaned four times on PBS including 0.2\% Triton X-100 (PBST).

Mouse monoclonal antibodies against TH (diluted $1: 2000$ in PBST with 10\%v/v normal horse serum; Santa Cruz Biotechnology, Inc., Dallas, TX, USA) were incubated with sections for 72 hours at $4^{\circ} \mathrm{C}$. Sections were incubated with biotinylated anti-mouse (Vector Laboratories, Inc., Burlingame, CA, USA) diluted 1 : 200 in PBST with 2\%v/v normal horse serum for 2 hours at room temperature after rinsing by PBST. These sections were incubated with avidin-biotin-peroxidase complex reagent (Vector Laboratories, Inc.) for 2 hours. Sections were developed through diaminobenzadine peroxidase substrate kit (Vector Laboratories, Inc.) after rinsing by PBST. These sections were mounted on slides and cover slipped for microscopic examination. Density of immunopositive neurons in the dorsal striatum (AP, +1.20 to +0.84 from bremga) was quantified according to published method of Paxinos ${ }^{23)}$ using Scion image program (Scion Corp., Frederick, MD, USA). For 6-HD categories (6-HD lesion and 6-HD lesion with DBS), only those rats that did not show TH-reactivity in the ipsilateral side of the striatum were included for the analysis of L-glutamate concentration.

\section{Cresyl violet staining}

At the end of microdialysis, coronal cryostat sections of $\mathrm{MFB}$, dorsal striatum, and GPi were stained to verify the site of 6-HD lesion and positions of probes and the electrode. Brain sections were mounted on gelatin-coated slides. These sections were rehydrated in $90 \%$ and $75 \%$ ethanol and distilled water for 2 minutes each. They were then stained with a cresyl violet solution for 5 minutes. They were dehydrated in ethanol concentrations and cover slipped with mountant.

\section{Statistical analysis}

Data are expressed as mean \pm standard error of mean (SEM). Repeated measures analysis of variance (ANOVA) was performed to find out the significance of any changes in HPLC analysis of glutamate result among different categories. Repeated measures ANOVA were based on six time points : 20 minutes before DBS, DBS start time (0 minute), DBS ending time (20 minutes), 40 minutes, 60 minutes, and 80 minutes after DBS start time. One-way ANOVA was performed to find out the significance of any changes in histochemical data 
among different categories followed by Tukey post hoc. Statistical method was done through MedCalc Statistical Software version 18.2.1 (MedCalc Software bvba, Ostend, Belgium; http://www.medcalc.org; 2018). The criterion for statistical significance was set at $p<0.05$.

\section{RESULTS}

\section{Influence of EPN stimulation on glutamate levels in striatum}

Results of mean $( \pm$ SEM) quantification of extracellular striatal glutamate levels in NL, NL with DBS, 6-HD, and 6-HD with DBS categories are shown in Figs. 1 and 2. As illustrated in Fig. 1, there was no statistical significance in extracellular striatal glutamate value between NL category and NL with DBS category $(p=0.513)$. However, there was statistically significant difference within categories $(p=0.016)$ and within interaction in category and time $(p=0.039)$. HPLC demonstrated a tendency of an increase in extracellular glutamate level at 20 minutes after DBS in NL with DBS category as contrast with NL category. The maximal peak of extracellular glutamate was demonstrated at 40 minutes following DBS onset in NL with DBS category. Extracellular glutamate level slowly declined to baseline value in 40 minutes after DBS ending time.

As illustrated in Fig. 2, there was no statistically significant difference in extracellular striatal glutamate value between 6 -HD category and 6-HD with DBS category ( $p=0.188$ ). However, there was statistical significance within categories $(p=0.032)$. There was no statistical significance within interaction of category and time $(p=0.118)$. HPLC analysis demonstrated a tendency of an increase in extracellular striatal glutamate level immediately after DBS in 6-HD with DBS category as contrast with 6-HD category. The maximal peak of extracellular glutamate was demonstrated at 20 minutes following DBS onset in 6-HD with DBS category. Extracellular glutamate level slowly declined to baseline value in 20 minutes after DBS ending time.

\section{Influence of EPN stimulation on TH-immunore- activity in striatum and $\mathrm{SN}$}

As illustrated in Figs. 3 and 4, dopamine depletion was severe in $\mathrm{SN}$ and striatum in 6-HD lesion categories. Optical density measurement of TH immunoreactivity in SN and striatum in 6-HD lesioned categories demonstrated a significant depletion as contrasted with NL categories $\left(\mathrm{F}_{3,23}=56.471\right.$
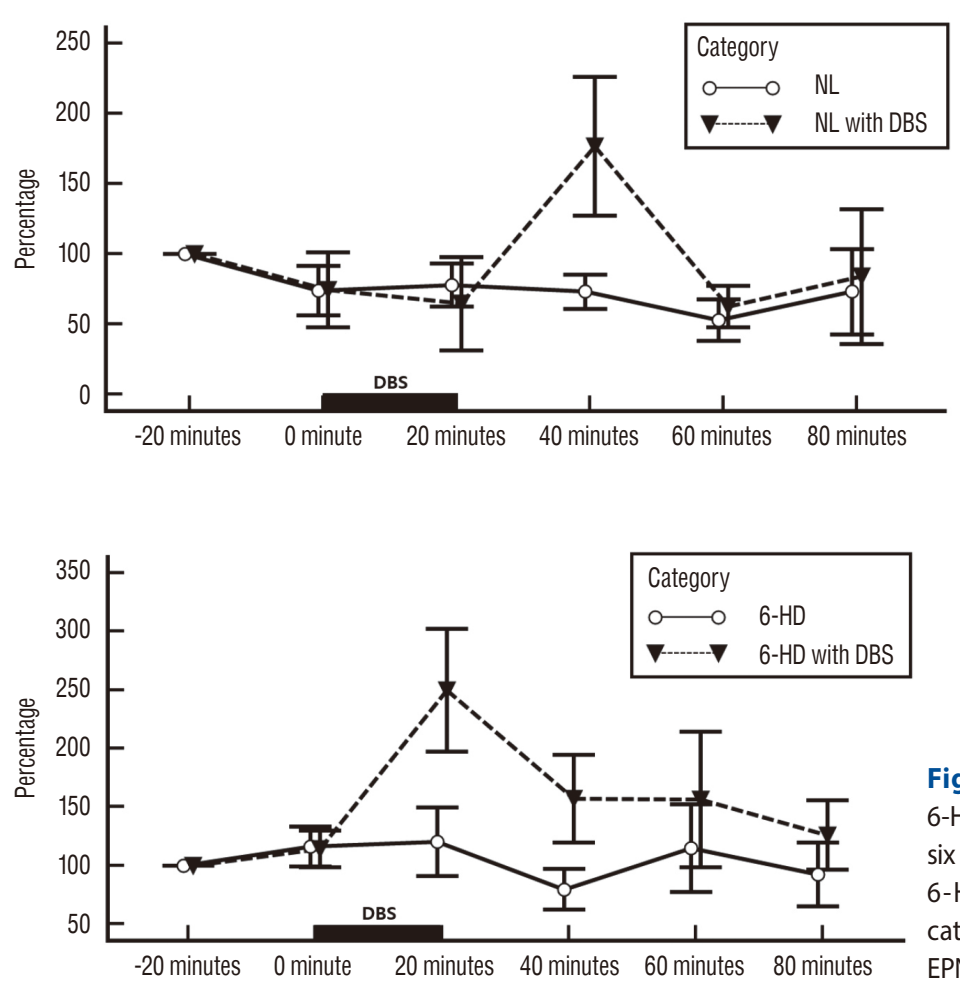

Fig. 1. Influence of EPN stimulation on striatal glutamate levels in no-lesioned rats. Each point represents the mean \pm standard error from six rats per category : no-lesioned rats without deep brain stimulation (NL category) and no-lesioned rats with deep brain stimulation (NL with DBS category). DBS : deep brain stimulation, $\mathrm{NL}$ : no-lesioned, EPN : entopeduncular nucleus.

Fig. 2. Influence of EPN stimulation on striatal glutamate levels in $6-\mathrm{HD}$ lesioned rats. Each point represents the mean \pm standard error from six rats per category : 6-HD lesioned rats without DBS (6-HD category) and 6-HD lesioned rats with deep brain stimulation (6-HD with DBS category). DBS : deep brain stimulation, 6-HD : 6-hydroxydopamine, EPN : entopeduncular nucleus. 

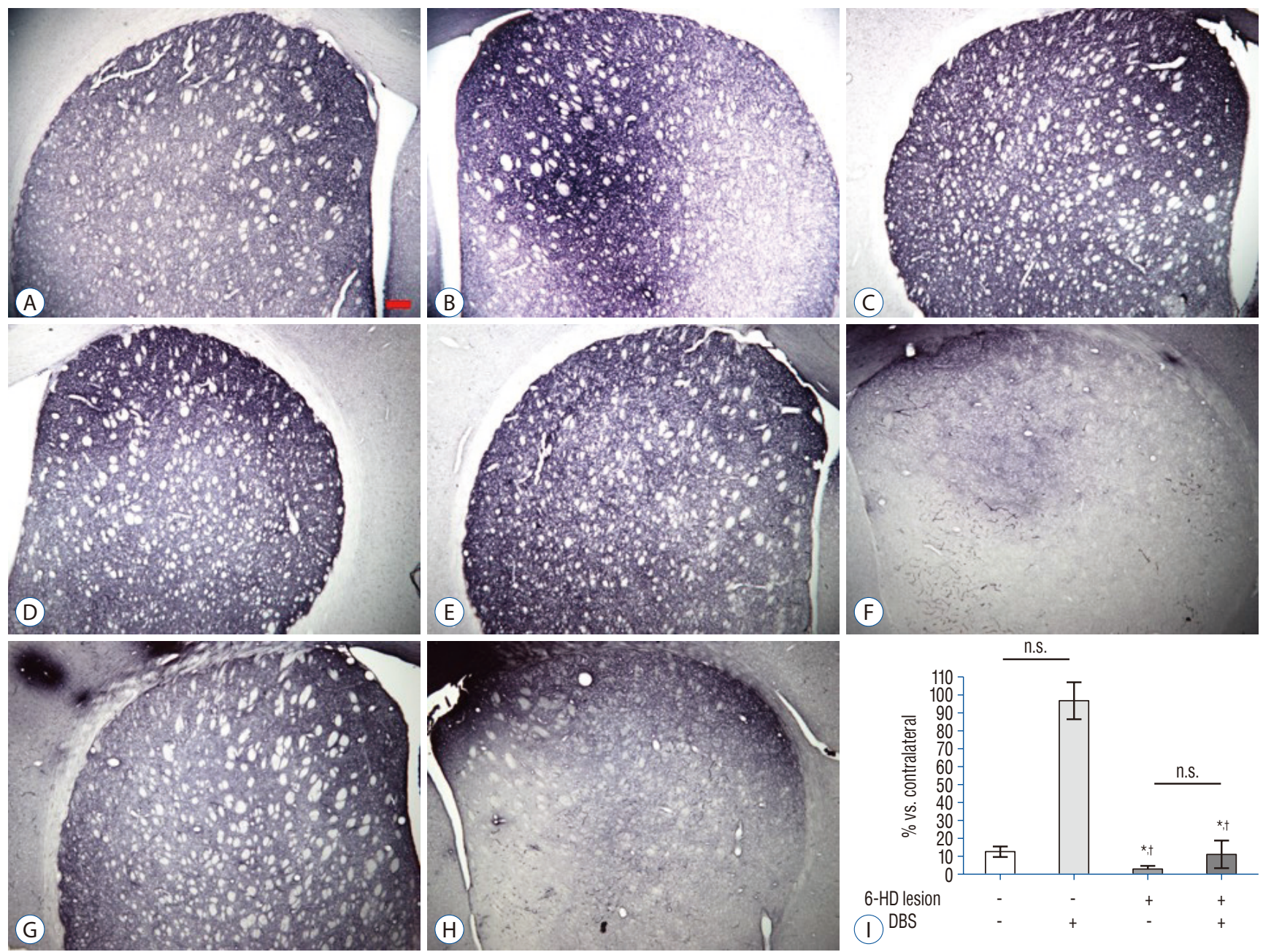

Fig. 3. TH-immunoreactivity in the Striatum. Representative images showed TH-immunoreactivity in the striatum of the ipsilateral (A, $C, E, G)$ and contralateral $(B, D, F, H)$ sides. Scale bar=200 $\mu \mathrm{m}$. A and B : NL category. C and D : NL with DBS category. E and F : 6-HD category. G and H : 6-HD with DBS category. Mean ( \pm standard error of mean) quantification (I) of TH expression showed significant decrease in both 6-HD and 6-HD with DBS categories compared to NL categories ( $\mathrm{n}=5-6$ per groups). ${ }^{*} p<0.001$ versus NL category. ${ }^{\dagger} p<0.001$ versus NL with DBS category, ANOVA. n.s. : no significance, $6-\mathrm{HD}$ :6-hydroxydopamine, DBS : deep brain stimulation, TH : tyrosine hydroxylase, NL : no-lesioned, ANOVA : analysis of variance.

and $\mathrm{F}_{3,18}=22.262$ in the striatum and $\mathrm{SN}$ respectively, $p<0.001$; Figs. 3 and 4). DBS had no statistically significant effect on the expression of TH in NL or 6-HD lesion category.

\section{Histologic verification}

At the end of the experiment, histologic evaluations revealed the exact location of 6-HD lesion tip in MFB (Fig. 5A and B), microdialysis cannula tip in striatum (Fig. 5C and D), and stimulating electrode tip in EPN (Fig. 5E and F) by cresyl violet staining.

\section{DISCUSSION}

GPi stimulation is a potent treatment of advanced PD. However, the mechanism by which GPi stimulation influences the basal ganglia circuit remains unclear.

Loss of dopaminergic innervation in SN compacta causes hyperactivity in the GPi, i.e., principal output of basal ganglia and a severe disruption in motor circuit. In the opinion of the DeLong theory, increase of inhibitory basal ganglia output appeared in PD is mostly attributable to hyperactivity of GPi and $\mathrm{STN}^{8)}$.

Output nuclei of the basal ganglia consist of SN reticulata 


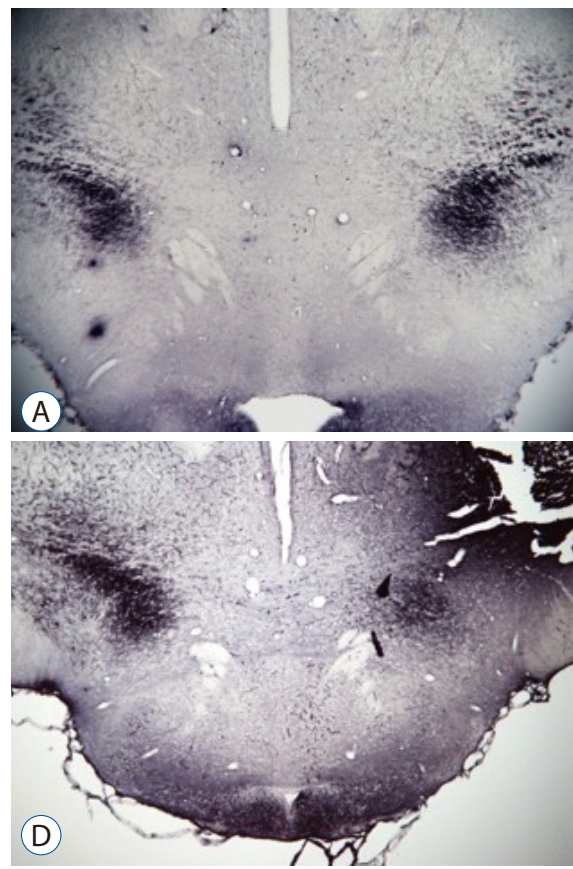

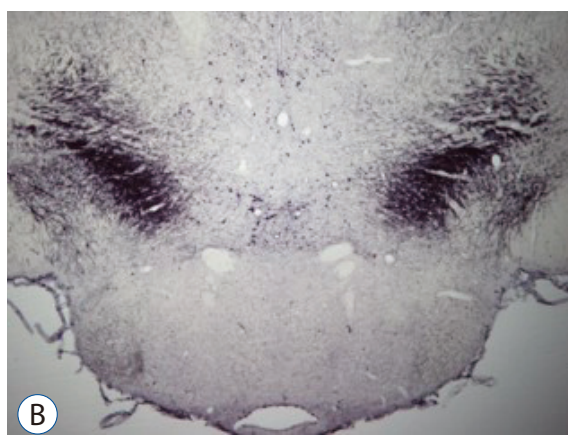

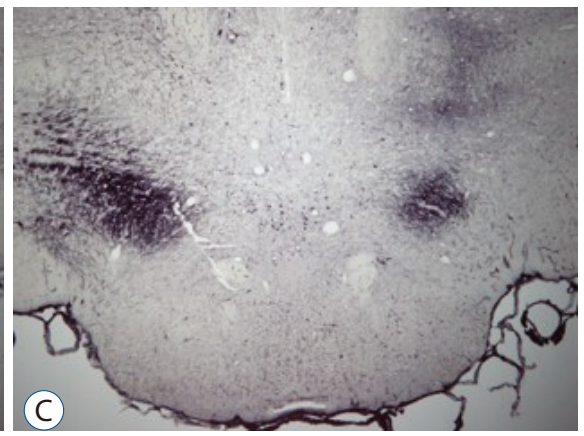

n.s.

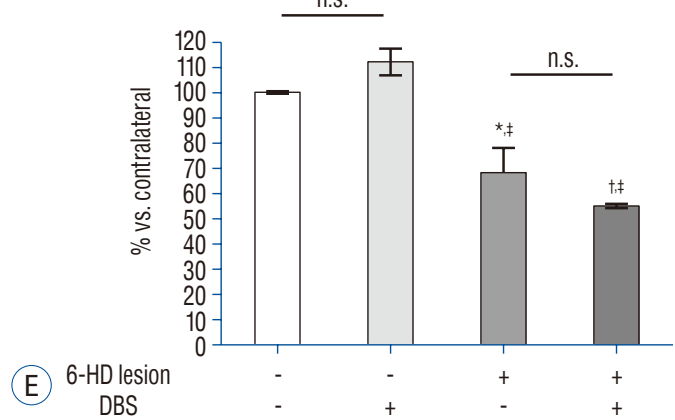

Fig. 4. TH-immunoreactivity in the SN. Representative images showed TH-immunoreactivity in the SN. Left side is ipsilateral part and right side is contralateral part of the SN. Scale bar=200 $\mu \mathrm{m}$. A : NL category. B : NL with DBS category. C : 6-HD category. D : 6-HD with DBS category. Mean ( \pm standard error of mean) quantification (E) of TH expression showed significant decrease in both 6-HD category and 6-HD with DBS category compared to NL categories ( $\mathrm{n}=4-6$ per groups). ${ }^{*} p<0.01$ and ${ }^{\dagger} p<0.001$ versus NL category. ${ }^{\ddagger} p<0.001$ versus NL with DBS category, ANOVA. n.s. : no significance, $6-\mathrm{HD}$ : 6-hydroxydopamine, DBS : deep brain stimulation, SN : substantia nigra, TH : tyrosine hydroxylase, NL : no-lesioned, ANOVA : analysis of variance.

(SNr) and GPi. GPi receives direct inhibitory pathway from striatum and indirect excitatory pathway from STN. Output neurons from GPi can project to centromedian, ventral anterior and ventral lateral nuclei of the thalamus. The STN plays a potent part in modulating output nuclei of basal ganglia (GPi and $\mathrm{SNr}$ ). It is an essential structure in indirect pathway of the basal ganglia ${ }^{8)}$. Ansa lenticularis and lenticular fasciculus are two major bundles from GPi to thalamus ${ }^{7}$.

It is well known that GPi has many GABAergic terminals. These greatly exceed the number of excitatory glutamatergic terminals on these neurons that arise from the $\mathrm{STN}^{10)}$. Thus, GPi stimulation will inhibit of basal ganglia output, i.e., reduce the release of GABA (inhibitory neurotransmitter). However, it is not known whether this is the therapeutic theory of GPi stimulation.

Up to date, there have been many studies about striatal glutamate changes associated with STN DBS ${ }^{2,5,6)}$. However, to the best of our knowledge, changes in striatal glutamate associated with GPi stimulation are currently unknown.

As mentioned above, the EPN of the rat corresponds to the GPi in primates and humans. It has the similar connections within the basal ganglia ${ }^{11}$. Many studies have found that high frequency stimulation is more effective than low frequency stimulation in STN and $\mathrm{GPi}^{4,17,28)}$. We also performed high frequency stimulation (frequency $130 \mathrm{~Hz}$ ) of EPN in this study.

We inserted stimulation electrode of EPN to all categories (NL category, NL with DBS category, 6-HD category, and 6-HD with DBS category) to rule out a microlesion effect. EPN stimulation increased striatal glutamate levels in NL with DBS category and 6-HD with DBS category compared to NL category and 6-HD category, respectively. This result is in accordance with findings of other reports about increased striatal glutamate levels after STN stimulation ${ }^{9,11,15)}$. Such same results of increased striatal glutamate in both STN and EPN stimulation cannot be explained by the same mechanism of STN and EPN stimulation. Our results are supported by the previous study that optogenetic inhibition of EPN in rat model changed thalamic output and improved Parkinsonian behaviors ${ }^{20)}$. Therefore, we suggest that GPi DBS decreases the inhibitory signal to the thalamus, which eventually increases thalamic output and striatal glutamate. Meissner et al. ${ }^{19)}$ previ- 

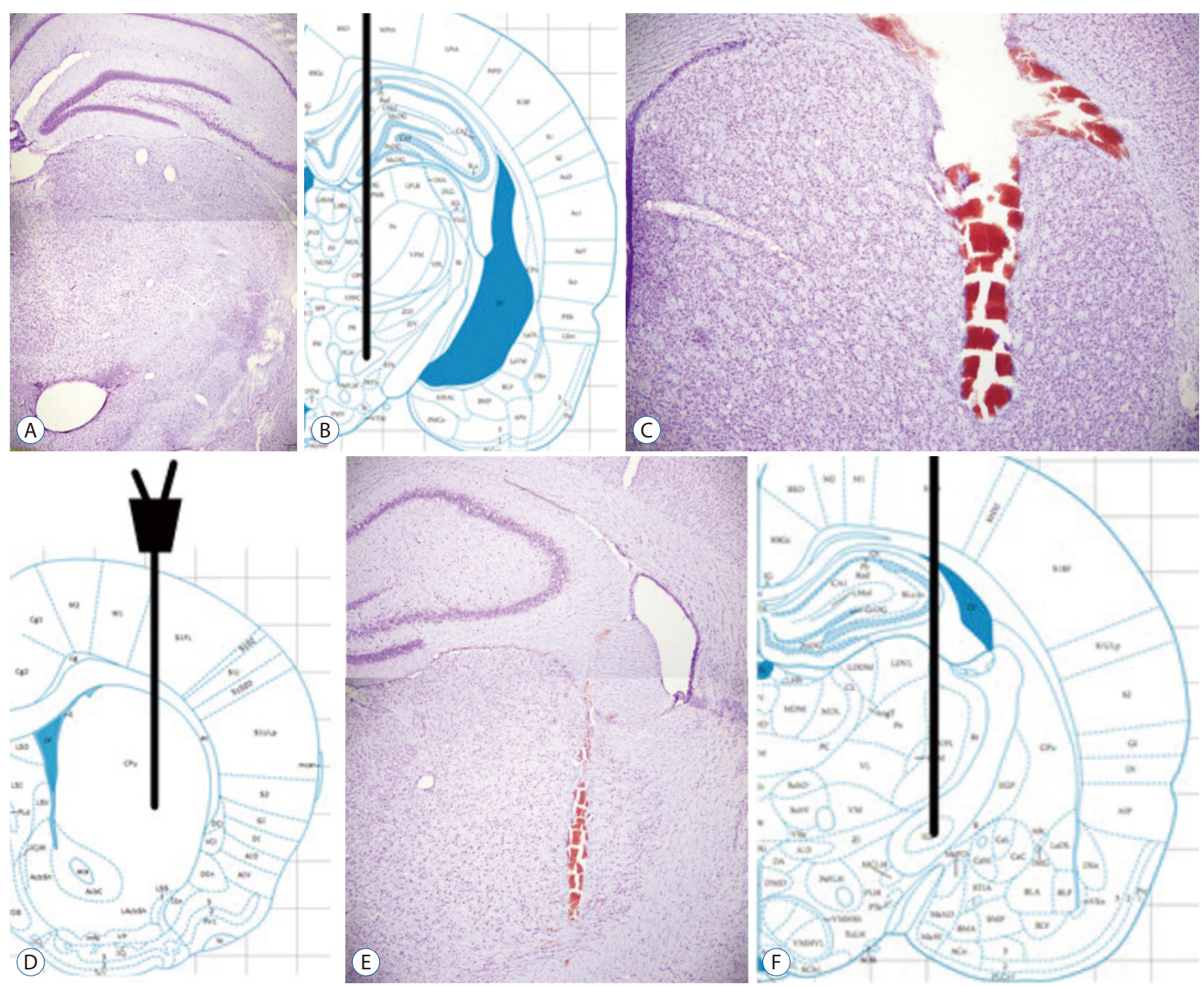

Fig. 5. Histological verification. The stereotaxic atlas from Paxinos ${ }^{23)}$ shows the location of MFB (B), dorsal striatum (D) and EPN (F). Representation of coronal sections verifies injection site of 6-hydroxydopamine in the MFB (A), position of the probe in the striatum (C) and the electrode in the EPN (E). Scale bars $=200 \mu \mathrm{m}$. MFB : medial forebrain bundle, EPN : entopeduncular nucleus.

ously reported that GPi DBS does not significantly influence the striatal dopamine. Abosch et al. ${ }^{1)}$ suggested that STN DBS in PD does not produce striatal dopamine release. On the other hand, Bruet et al. ${ }^{6}$ suggested that STN DBS influences glutamate and GABA in striatum and that this result is modulated by dopamine receptor antagonist. As far as we know, relationships between the changes of dopamine and glutamate in striatum associated with GPi DBS remain unclear. Although we cannot explain whether our results influence striatal dopamine, we assume that striatal glutamate is bound up with GPi DBS for PD compared to striatal dopamine. Further studies are needed to clarify relationships between the changes of dopamine and glutamate in striatum associated with GPi DBS.

In terms of levodopa-induced dyskinesia, effective STN DBS produced an increase of cGMP within the GPi, the marker of increase glutamatergic transmission ${ }^{26)}$. On the other hand, our results are that GPi DBS produced an increase of glutamate within the striatum, but not within the GPi. This difference may reflect the antidyskinetic effect of GPi DBS compared to STN DBS. However, the limitation of our study is that we cannot explain the direct antidyskinetic effect of GPi DBS by only this difference of an increase of glutamate in striatum versus $\mathrm{GP}^{24)}$. 
Clinically, GPi and STN DBS can significantly improve advanced PD symptoms. However, advantages of STN and GPi stimulation are known to be different. GPi DBS allows greater recovery of verbalized fluency and depression symptoms while STN DBS is associated with decreased dosage of levodopa equivalent ${ }^{16,27}$. Increased striatal glutamate does little to truly explain such a discrepancy in advantages between STN and GPi stimulations. Further investigation is required to prove the reason for such difference. These results suggest the view that the impact of GPi DBS on pathophysiological function of BG is not only due to changes in GPi outflow, but also due to complex mechanisms in BG circuit.

The increase in striatal glutamate estimated during EPN DBS in NL and NL with DBS category and in 6-HD and 6-HD with DBS category can be clarified by increase in activity of excitatory glutamatergic afferents in striatum. The inhibition of activity of BG outputs caused by GPi DBS could cause disinhibition of thalamic-cortical pathway, thus activating cortico-striatal pathway and thalamic-striatal pathway.

Finally, we provided evidence that GPi DBS might play an essential role in enhancing decreased activity of excitatory input from cortex to striatum. Our results suggest that reinforcement of cortico-striatal pathway can be mediated by GPi DBS.

It was of interest that earlier reaction of glutamate change was made at 6-HD with DBS category compared to NL with DBS category. The reason of this finding might be sensitivity differences of striatal glutamatergic pathway to GPi stimulation between NL and 6-HD categories. In case of 6-HD with DBS category, the rapid reaction of glutamate change reflects therapeutic advantage of EPN DBS. In case of NL with DBS category, EPN DBS seems to make a slow response of glutamate level under physiologic basal ganglia circuit. To our knowledge, there has been no evidence of these finding. Further investigations are needed to prove sensitivity differences of striatal glutamate between 6-HD with DBS category and NL with DBS category.

\section{Limitation}

The main limitations of our study included stimulation of EPN under anesthesia that was performed on rats not freely moving, short-term, and only one time of stimulation of EPN that could not correspond to continuous stimulation.

\section{CONCLUSION}

Clinical outcomes of GPi DBS are not only limited to straight GPi. They also include extensive alteration within basal ganglia.

\section{CONFLICTS OF INTEREST}

No potential conflict of interest relevant to this article was reported.

\section{- Acknowledgements}

This research was supported by Basic Science Research Program through the National Research Foundation of Korea (NRF) funded by the Ministry of Education, Science and Technology (NRF-2014R1A1A1A05007768). The authors wish to acknowledge the financial support of the Catholic Medical Center Research Foundation made in the program year of 2018.

\section{References}

1. Abosch A, Kapur S, Lang AE, Hussey D, Sime E, Miyasaki H, et al. : Stimulation of the subthalamic nucleus in Parkinson's disease does not produce striatal dopamine release. Neurosurgery 53 : 1095-1102; discussion 1102-1105, 2003

2. Agnesi F, Blaha CD, Lin J, Lee KH : Local glutamate release in the rat ventral lateral thalamus evoked by high-frequency stimulation. J Neural Eng $7: 26009,2010$

3. Bar-Gad I, Elias S, Vaadia E, Bergman H : Complex locking rather than complete cessation of neuronal activity in the globus pallidus of a 1-methyl-4-phenyl-1,2,3,6-tetrahydropyridine-treated primate in response to pallidal microstimulation. J Neurosci 24 : 7410-7419, 2004

4. Barnéoud $P$, Parmentier $S$, Mazadier $M$, Miquet JM, Boireau $A$, Dubédat $P$, et al. : Effects of complete and partial lesions of the dopaminergic mesotelencephalic system on skilled forelimb use in the rat. Neuroscience 67 : 837-848, 1995

5. Boulet $S$, Lacombe E, Carcenac C, Feuerstein C, Sgambato-Faure V, Poupard A, et al. : Subthalamic stimulation-induced forelimb dyskinesias are linked to an increase in glutamate levels in the substantia nigra pars reticulata. J Neurosci 26 : 10768-10776, 2006

6. Bruet N, Windels F, Carcenac C, Feuerstein C, Bertrand A, Poupard A, et al. : Neurochemical mechanisms induced by high frequency stimulation of the subthalamic nucleus: increase of extracellular striatal glutamate 
and GABA in normal and hemiparkinsonian rats. J Neuropathol Exp Neurol 62 : 1228-1240, 2003

7. DeLong MR : Primate models of movement disorders of basal ganglia origin. Trends Neurosci $13: 281-285,1990$

8. DeLong MR, Wichmann $T$ : Basal ganglia circuits as targets for neuromodulation in Parkinson disease. JAMA Neurol 72 : 1354-1360, 2015

9. Gubellini P, Eusebio A, Oueslati A, Melon C, Kerkerian-Le Goff L, Salin $P$ : Chronic high-frequency stimulation of the subthalamic nucleus and L-DOPA treatment in experimental parkinsonism: effects on motor behaviour and striatal glutamate transmission. Eur J Neurosci 24 : 18021814, 2006

10. Hazrati LN, Parent $A$ : Differential patterns of arborization of striatal and subthalamic fibers in the two pallidal segments in primates. Brain Res $598: 311-315,1992$

11. Jonkers N, Sarre S, Ebinger G, Michotte Y : MK801 suppresses the LDOPA-induced increase of glutamate in striatum of hemi-Parkinson rats. Brain Res 926 : 149-155, 2002

12. Lanciego JL, Gonzalo N, Castle M, Sanchez-Escobar C, Aymerich MS, Obeso JA : Thalamic innervation of striatal and subthalamic neurons projecting to the rat entopeduncular nucleus. Eur J Neurosci 19 : 1267-1277, 2004

13. Lang $A E$, Widner $H$ : Deep brain stimulation for Parkinson's disease: patient selection and evaluation. Mov Disord 17 Suppl 3 : S94-S101, 2002

14. Lee KH, Kristic K, van Hoff R, Hitti FL, Blaha C, Harris B, et al. : Highfrequency stimulation of the subthalamic nucleus increases glutamate in the subthalamic nucleus of rats as demonstrated by in vivo enzymelinked glutamate sensor. Brain Res 1162 : 121-129, 2007

15. Lee KJ, Shim I, Sung JH, Hong JT, Kim IS, Cho CB : Striatal glutamate and GABA after high frequency subthalamic stimulation in parkinsonian rat.

J Korean Neurosurg Soc 60 : 138-145, 2017

16. Liu Y, Li W, Tan C, Liu X, Wang X, Gui Y, et al. : Meta-analysis comparing deep brain stimulation of the globus pallidus and subthalamic nucleus to treat advanced Parkinson disease. J Neurosurg 121 : 709-718, 2014

17. McConnell GC, So RQ, Hilliard JD, Lopomo P, Grill WM : Effective deep brain stimulation suppresses low-frequency network oscillations in the basal ganglia by regularizing neural firing patterns. J Neurosci 32 : 15657-15668, 2012
18. McIntyre CC, Savasta M, Kerkerian-Le Goff L, Vitek JL : Uncovering the mechanism(s) of action of deep brain stimulation: activation, inhibition, or both. Clin Neurophysiol 115 : 1239-1248, 2004

19. Meissner W, Paul G, Reum T, Reese R, Sohr R, Morgenstern R, et al. : The influence of pallidal deep brain stimulation on striatal dopaminergic metabolism in the rat. Neurosci Lett 296 : 149-152, 2000

20. Moon HC, Won SY, Kim EG, Kim HK, Cho CB, Park YS : Effect of optogenetic modulation on entopeduncular input affects thalamic discharge and behavior in an AAV2- $\alpha$-synuclein-induced hemiparkinson rat model. Neurosci Lett 662 : 129-135, 2018

21. Odekerken VJ, van Laar T, Staal MJ, Mosch A, Hoffmann CF, Nijssen PC, et al. : Subthalamic nucleus versus globus pallidus bilateral deep brain stimulation for advanced Parkinson's disease (NSTAPS study): a randomised controlled trial. Lancet Neurol 12 : 37-44, 2013

22. Papa SM, Engber TM, Kask AM, Chase TN : Motor fluctuations in levodopa treated parkinsonian rats: relation to lesion extent and treatment duration. Brain Res 662 : 69-74, 1994

23. Paxinos $G$ : The rat nervous system, ed 3. San Diego : Elsevier Academic Press, 2004, pp49-56

24. Sgambato-Faure V, Cenci MA : Glutamatergic mechanisms in the dyskinesias induced by pharmacological dopamine replacement and deep brain stimulation for the treatment of Parkinson's disease. Prog Neurobiol $96: 69-86,2012$

25. Shipton EA : Movement disorders and neuromodulation. Neurol Res Int 2012 : 309431, 2012

26. Stefani A, Fedele E, Galati S, Pepicelli O, Frasca S, Pierantozzi M, et al. : Subthalamic stimulation activates internal pallidus: evidence from cGMP microdialysis in PD patients. Ann Neurol 57 : 448-452, 2005

27. Tan ZG, Zhou Q, Huang T, Jiang Y : Efficacies of globus pallidus stimulation and subthalamic nucleus stimulation for advanced Parkinson's disease: a meta-analysis of randomized controlled trials. Clin Interv Aging 11 : 777-786, 2016

28. Temel Y, Visser-Vandewalle V, Aendekerk B, Rutten B, Tan S, Scholtissen $B$, et al. : Acute and separate modulation of motor and cognitive performance in parkinsonian rats by bilateral stimulation of the subthalamic nucleus. Exp Neurol 193 : 43-52, 2005

29. Wichmann T, Delong MR : Deep-brain stimulation for basal ganglia disorders. Basal Ganglia $1: 65-77,2011$ 